\title{
Research on recognition method of cloud precipitation particle shape based on BP neural network
}

\author{
Haonan Dong ${ }^{1}$, Ruili $\mathrm{Jiao}^{1,{ }^{*}}$, and Minsong Huang ${ }^{2}$ \\ ${ }^{1}$ Beijing Information Science and Technology University Information and Communication Technology \\ School, Beijing 100101, China \\ ${ }^{2}$ Key Laboratory of Cloud-Precipitation Physics and Severe Storms, Institute of Atmospheric Physics, \\ Chinese Academy of Sciences, Beijing 100029, China
}

Keywords: Cloud imaging probe, Automatic identification, Cloud particle habit, Habit identification, BP neural network, Classification model, Holroyd method, Accuracy.

\begin{abstract}
In order to solve the problem that the shape of cloud particle images measured by airborne cloud imaging probe (CIP) cannot be automatically recognized, this paper proposes an automatic recognition method of cloud and precipitation particle shape based on BP neural network. This method mainly uses a set of geometric parameters which can better describe the shape characteristics of cloud precipitation particles. Based on the cloud precipitation particle images measured by CIP in the precipitation stratiform clouds in northern China, a particle shape data training set and a testing set were constructed to train and verify the effect of the selected BP neural network model. The selected BP neural network model can classify the cloud particle image into tiny, column, needle, dendrite, aggregate, graupel, sphere, hexagonal and irregular. Utilizing the field campaign data measured by CIP, the habit identified results by the improved Holroyd method and by the selected BP neural network model were compared, which shows that the accuracy of BP neural network method is better than that of improved Holroyd method.
\end{abstract}

\section{Introduction}

The recognition of cloud precipitation particle shape is very important for many aspects of cloud microphysics research. The shape of cloud particles can affect their own scattering properties, growth rate and end-of-fall velocity. The scattering properties of cloud particles have an important impact on global climate, radiation balance, and satellite remote sensing. Although the calculation of the scattering properties of non-spherical particles is still one of the difficult problems in atmospheric radiation calculations (Guo Caili, 2005) ${ }^{[1]}$, the prerequisite for accurately calculating the scattering properties of cloud particles is still the

\footnotetext{
*Corresponding author: jiaoruili@bistu.edu.cn
} 
determination of the shape of cloud particles (Chen Qi, 2018) ${ }^{[2]}$. Therefore, the shape judgment of cloud particles is very important for cloud microphysics research.

At present, the main method of measuring cloud particles is that the airplane carries the cloud precipitation particle imager directly into the cloud for measurement. Among the particle measuring instruments carried by the aircraft, the cloud precipitation particle imager based on the photodiode array is one of the most widely used airborne cloud microphysical measuring instruments. Because this instrument can not only measure the size of cloud precipitation particles, but also record particle images, which can be used to identify particle shapes. Unfortunately, the instrument only records particle images, and the specific particle shape recognition work still needs to be done manually. The particle image of each aerial survey is basically measured in units of 10,000 , which is very difficult for manual recognition. Fortunately, the development of modern computer technology and image processing technology has made it possible for computers to replace humans. Therefore, the development of a computer-based automatic particle shape recognition algorithm has become the core work of particle shape recognition.

In the research of cloud precipitation particle shape recognition algorithm, Rahman et al. (1981) used adaptive Kalman filter method and Bayesian decision theory to classify cloud particle images into 7 categories based on cloud particle images collected by 2DC probes ${ }^{[3}$, 4]. Hunter et al. (1984) used the empirical orthogonal function to extract the feature values of the particle images, screened the feature values of the particles through the ADAPT algorithm, and linked the screened feature values with six particle shape types. The results show that machine recognition is more efficient and consistent than manual recognition methods ${ }^{[5]}$. Holroyd (1987) used the geometric feature parameters of the particle image to identify the shape of the particles in the image measured by the 2DC probe, and finally divided the shape of the cloud particles into 9 categories ${ }^{[6]}$. Duroure et al. (1994) proposed the concept of "particle swarm", which can effectively improve the calculation speed and the accuracy of the corresponding parameters by using the geometric characteristics and probability statistics of particles ${ }^{[7]}$. Korolev and Sussman (2000) used the particle characteristics and simple dimensionless ratio relationship based on the cloud particle images collected by the 2DC probe and divided the particle shapes into 4 categories by solving the inverse problem ${ }^{[8]}$. Lawson et al. (2006) used the simple geometric dimensions of particles to perform combination operations and finally divided the particle shapes measured by the CPI imager into 9 categories ${ }^{[9]}$. Lindqvist et al. (2012) used the principal component analysis method to divide the particle shape measured by the CPI imager into 8 categories ${ }^{[10]}$.

In China, the recognition of cloud precipitation particle shape started late, and there are few related studies. Sun Haiyang et al. (2014) proposed a method for cloud particle shape classification using moments and Fourier descriptors ${ }^{[11]}$. Wang Lei et al. (2014) proposed a particle shape recognition method suitable for gray-scale probes. The proposed method can divide the two-dimensional image of particles into 8 categories ${ }^{[12]}$. Huang Minsong et al. (2020) proposed an improved Holroyd cloud particle shape recognition method, and finally cloud particle images can be divided into 8 categories ${ }^{[13]}$. It can be seen that the existing methods are all based on the threshold recognition process, but this method inevitably has certain defects in the recognition process, that is, the cloud particles measured by different probes and different regional environments need to select different particle shape recognition thresholds. The artificially specified threshold may cause inaccurate division of results, and there will be certain errors. However, the use of BP neural network and the advantages of its algorithm's own good learning ability and adaptability can better overcome the defects of the threshold-based classification method. Secondly, due to the different number of light array elements and light array units contained in different imaging instruments, the imaging optical system parameter settings of the instruments are also different, resulting in different resolutions and measurement ranges of the instruments, so the particle shape recognition 
method corresponding to each type of airborne cloud precipitation particle imager is different. At present, one of the main photoelectric array imaging instruments on Chinese cloud microphysical detection aircraft is the cloud imaging probe (CIP) developed by the US Particle Measurement Technology (DMT). Therefore, it is necessary to develop a CIP instrument that can measure it. The method of automatically identifying the shape of cloud precipitation particle images is used to assist China's research on cloud precipitation physics and artificial weather modification.

\section{Cloud particle shape categories}

\subsection{CIP instrument laser measurement principle}

The cloud imaging probe (CIP) developed by DMT is a kind of imaging array detector. Its sensing element is composed of 64-unit photodiode array, and the sampling space is composed of a collimator and light intensity. The laser beam is composed of a relatively uniform distribution. When a particle passes through, it will project the particle onto the sensor element. The current value generated by the sensor unit changes due to the shadow of the particle. The condition for setting the photodiode to be blocked is that the received light energy attenuates more than $50 \%$. When any unit meets the light energy attenuation threshold, the electronic circuit is used to detect the current of each photodiode on the sensor at a certain frequency and process the detected current. The signal obtained during each processing is called 1 slice. When multiple slice signals obtained by sampling are spliced together in the order of acquisition, a binary image of the particle can be reconstructed.

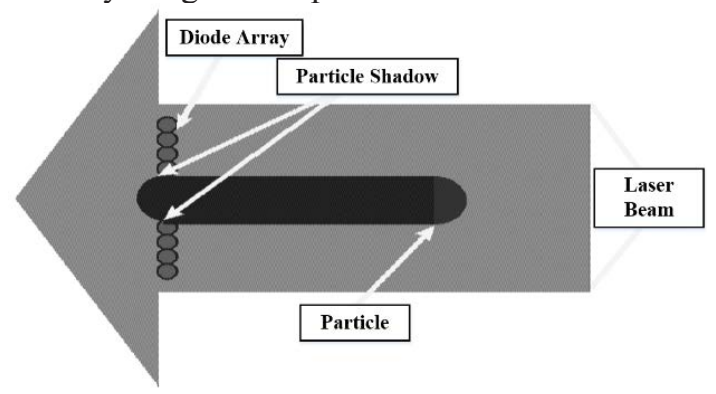

Fig. 1. Particle imaging with CIP probe.

\subsection{Cloud particle shape classification}

Due to the ever-changing shapes of cloud particles in nature, it is a very difficult task to classify the shape of cloud particles. Different researchers have put forward different shape classifications for different instruments and research needs. Nakaya (1954) divided cloud particle shapes into 41 categories, Zamorsky (1955) divided them into 72 categories, Magono and Lee (1966) supplemented the original shape classification and proposed 80 types of particle shape classification. Korolev et al. (2000) classified ice crystal particle shapes into 4 categories. Holroyd (1987) divided particle shapes into 8 categories, while Korolev et al. (2000) divided particle shapes into 4 categories.

The research basis of this paper is based on the cloud imaging probe (CIP). Because the pixel resolution of CIP is low $(25 \mu \mathrm{m})$, the pixel gray level (only black and white two gray levels), the determination of the number of particle shape categories must consider the instrument used for the image measurement and the purpose of the image. Therefore, in summary, this article mainly draws on the characteristic parameters of Holroyd and expands 
it appropriately. The cloud particles are divided into 9 shapes, which are tiny, column, dendrite, aggregate, graupel, irregular, needle, hexagonal and sphere. Examples of each shape classification are shown in Figure 2.

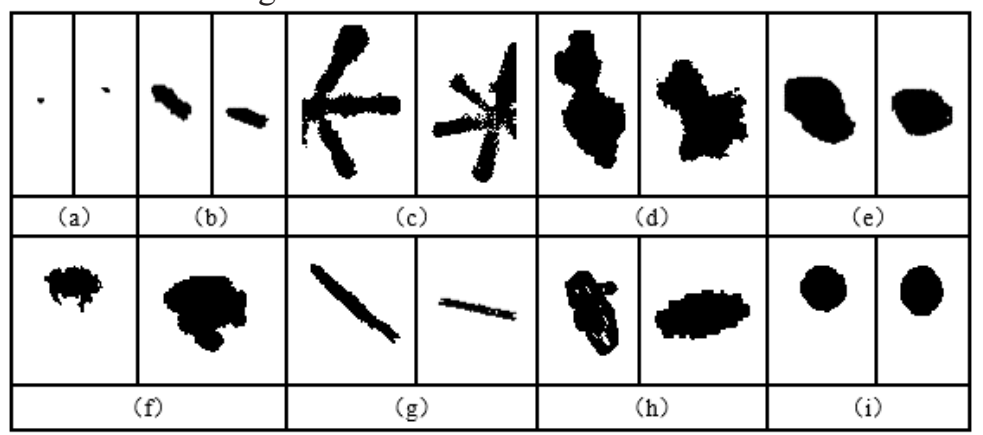

Fig. 2. Examples of nine kinds of particle shape images.

(a. tiny; b. column; c. dendrite; d. aggregate; e. graupel; f. irregular; g. needle; h. hexagonal; i. sphere)

\section{Cloud particle shape recognition method}

\subsection{Holroyd characteristic parameters for cloud particle shape recognition}

The feature parameters used in the BP neural network algorithm for identifying the shape of cloud particles proposed in this paper are partly based on the Holroyd (1987) method, and on this basis, new feature parameters are improved and expanded. The Holroyd feature parameters are shown in Figure 3. In the figure, $x$ is the number of particle pixels in the flight direction of the aircraft; $y$ is the number of particle pixels in the optical array direction; $N_{x}$ is the maximum number of pixels in the aircraft flight direction; $N_{y}$ is the maximum number of pixels in the optical array direction; $d$ is the particle span length (number of pixels) of the pixel in the direction obtained by the least squares fitting with the $x$ direction as the abscissa and the $y$ direction as the ordinate; $w$ is the particle width in the vertical direction of $d$ (the number of pixels); $r$ is the correlation coefficient of the image pixels in the $x$ and $y$ directions; $A$ is the direction angle of the particles. In addition, there are some key parameters not marked in the figure, where $a$ is the area of the particle (unit is the number of pixels); $P$ is the perimeter of the particle (unit is the number of pixels); $F$ is a specific geometric parameter, set as: $F=P d / a$ (dimensionless quantity); $N_{\text {total }}$ is the number of pixels of all particles, $N_{\text {edge }}$ is the number of pixels on the left and right edges of the plane's flight direction. $S$ is a parameter used to identify the shape of graupel particles, which is calculated as the ratio of the number of pixels that continuously fill the entire line of the light array in the $x$ direction to $N_{\text {total }}$.

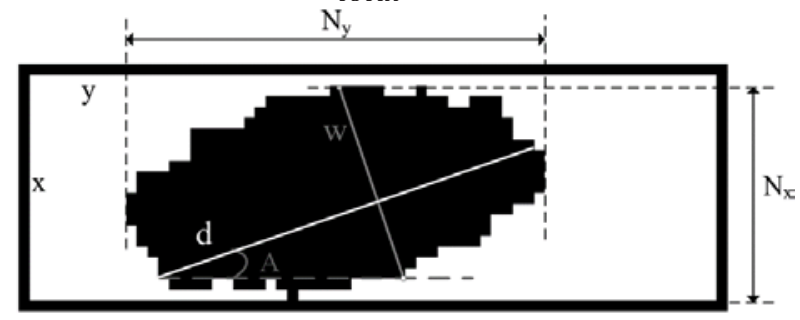

Fig. 3. Parameters of particle habit features in the Holroyd method. 


\subsection{Other characteristic parameters for cloud particle shape recognition}

(1) Circular degree: $e=4 \pi * a / P^{2}$, where $a$ is the area of the cloud particle image, $P$ is the perimeter of the cloud particle image.

(2) Convexity Area(CA): $C A=a / t u b a o A$, where $a$ is the area of the cloud particle image, tubaoA is the area of the convex hull.

(3) Convexity Perimeter(CP): $C P=P /$ tubaoP, where $P$ is the perimeter of the cloud particle image, tubaoP is the perimeter of the convex hull.

(4) Rectangularity(R): $R=a /\left(N_{x} * N_{y}\right)$, where $a$ is the area of the cloud particle image, $N_{x}$ is the maximum number of particle pixels in the $x$ direction, $N_{y}$ is the maximum number of particle pixels in the $y$ direction.

(5) Form Parameter(FP): $F=a / P$, where $a$ is the area of the cloud particle image, $P$ is the perimeter of the cloud particle image.

(6) Axis Ratio(AR): $A R=N_{y} / N_{x}$, where $N_{y}$ is the maximum number of particle pixels in the $y$ direction; $N_{x}$ is the maximum number of particle pixels in the $x$ direction.

\subsection{BP Neural Network Recognition Model of Cloud Particle Shape}

BP neural network is a multi-layer feedforward neural network trained according to the error back propagation algorithm. The topology is shown in Figure 4. As shown in the figure, in the BP neural network, the network structure is composed of multiple layers, each layer is connected by full connection, and the same layer is not connected to each other.

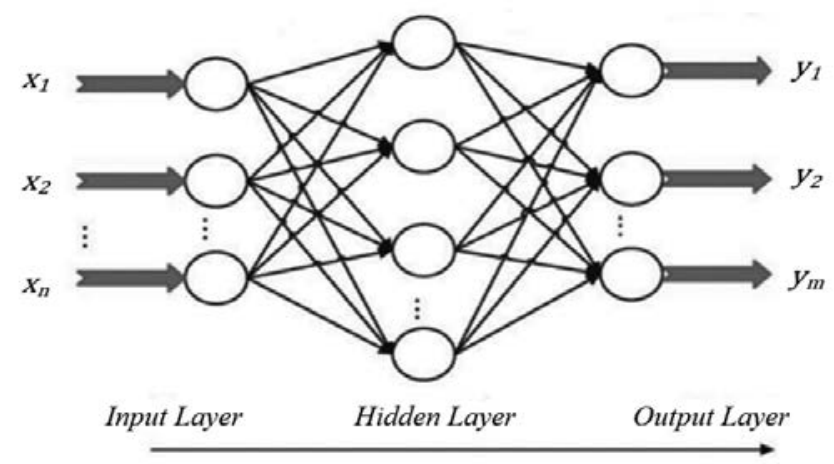

Fig. 4. BP neural network model.

Suppose that in the BP neural network, there are $n$ neurons in the input layer, $l$ neurons in the hidden layer, and $m$ neurons in the output layer. The connection weight from the $i$ th neuron in the input layer to the $j$-th neuron in the hidden layer is $w_{i j}$, and the deviation is $a_{j}$; the connection weight from the $j$-th neuron in the hidden layer to the $k$-th neuron in the output layer is $w_{j k}$, the deviation is $b_{k}$. The learning rate is $\eta$, the number of iterations is epochs, and the error threshold is $E_{0}$. The process iteration steps of BP neural network are as follows:

Step1: Initialize network parameters. Setting parameters: $n, l, m, \eta$, epocषss, $E_{0}$. The excitation function $f(x)$ is the Sigmoid function, which is determined by the following formula:

$$
\mathrm{f}(\mathrm{x})=\frac{1}{1+\mathrm{e}^{-\mathrm{x}}}
$$

Step2: Set the output $H_{j}$ of the $j$-th neuron of the hidden layer: 


$$
H_{j}=f\left(\sum_{i=1}^{n} w_{i j} x_{i}+a_{j}\right)
$$

Step 3: Calculate the output $y_{k}$ of the $k$-th neuron in the output layer:

$$
y_{k}=\sum_{j=1}^{l} H_{j} w_{j k}+b_{k}
$$

Step 4: Calculate the error $E$, suppose the ideal output of the current sample is $\hat{y}_{k}$, then the error $E$ from the actual output $y_{k}$ is: where

$$
E=\frac{1}{2} \sum_{k=1}^{m}\left(\hat{y}_{k}-y_{k}\right)^{2}=\frac{1}{2} \sum_{k=1}^{m} e_{k}^{2}
$$

where $e_{k}=\hat{y}_{k}-y_{k}$

Step 5: Weight update, you can use the following update formula to update the weights $w_{i j}, w_{j k}$ :

$$
\left\{\begin{array}{c}
w_{i j}=w_{i j}+\eta H_{j}\left(1-H_{j}\right) x_{i} \sum_{k=1}^{m} w_{j k} e_{k} \\
w_{j k}=w_{j k}+\eta H_{j} e_{k}
\end{array}\right.
$$

Step 6: Bias update, you can use the following update formula:

$$
\left\{\begin{array}{c}
a_{j}=a_{j}+\eta H_{j}\left(1-H_{j}\right) \sum_{k=1}^{m} w_{j k} e_{k} \\
b_{k}=w_{k}+\eta e_{k}
\end{array}\right.
$$

Step7: Determine whether the algorithm iteration is over, according to whether the error $E$ reaches the error threshold $E_{0}$ or whether the number of iterations Epochs reaches the maximum number of iterations, thereby judging whether the training is terminated.

This paper uses BP neural network to automatically recognize and classify the shape of cloud particles, which includes a learning process, a recognition and classification process. The learning process is to repeatedly train cloud particle image samples, continuously adjust network parameters, and finally determine a BP neural network classifier that can distinguish different cloud particle shapes; The recognition and classification process is based on the BP neural network classifier, which can automatically recognize and classify the decompressed single cloud particle image and give the recognition result. Therefore, the BP neural network classification design scheme for cloud particles is shown in Figure 5. The preprocessing part of cloud particle image data in the figure is mainly for the process of decompressing and filtering out false particles of CIP data files. Regarding the filtering of pseudo-particles, it mainly refers to the pseudo-particle identification method proposed by Huang Minsong et al. (2017) to filter out the subsequent BP neural network for cloud particle shape recognition.

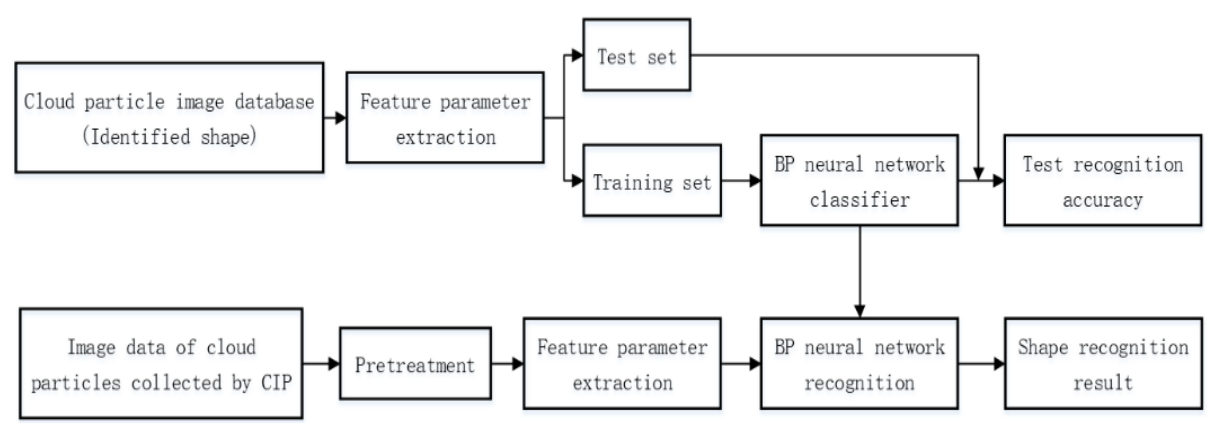

Fig. 5. BP neural network shape classification and recognition model for cloud particle image.

For BP neural network design, the main process is divided into three steps: The first step 
is the initialization of model parameters. The second step is to train the BP neural network. According to the cloud particle image data of the training set and the result of observing the shape of the cloud particles with the naked eye, according to the BP neural network process, the neural network related parameters are trained through continuous iteration. The third step is to test the BP neural network, use the trained BP neural network to test the cloud particle image data, and compare the results of the cloud particle shape with the naked eye, and give the BP neural network performance and accuracy.

The setting of the initialization parameters of the BP neural network is extremely important for the entire BP neural network model. Therefore, the following is a brief introduction to the determination method of parameter initialization.

This paper uses a 3-layer BP neural network structure, in which the hidden layer uses a nonlinear Sigmoid function, the output layer uses a linear function, and the training function uses a trainlm function.

Using Holroyd parameters and other relevant shape feature parameters as input data, that is, $n=22$ (the number of neurons in the input layer), and the number of nodes in the output layer corresponds to the number of cloud particle shape classifications (the tiny cloud particles are not considered here), that is, $m=8$ (the number of neurons in the output layer), the hidden layer determines the optimal number of nodes $l$ through trial and error. In this paper, $l$ takes 10 (the number of neurons in the hidden layer).

\subsection{BP neural network training and recognition results of particle shape}

For the classification of cloud particle shapes, this article divides them into 9 categories: tiny, column, dendrite, aggregate, graupel, irregular, needle, hexagonal and sphere. Among them, according to Holroyd (1987), we automatically classify a single cloud particle image with a total pixel less than 25 as tiny particles, which are not processed in the BP neural network. The remaining 8 types of cloud particle images are identified by BP neural network. This paper selects 8 types of cloud particle shape image samples, 80 training set samples for each type, 50 test set samples, a total of 1040 cloud particle images, and a simulation test based on Matlab software. The simulation test results are shown in Figure 6 and Table 1:

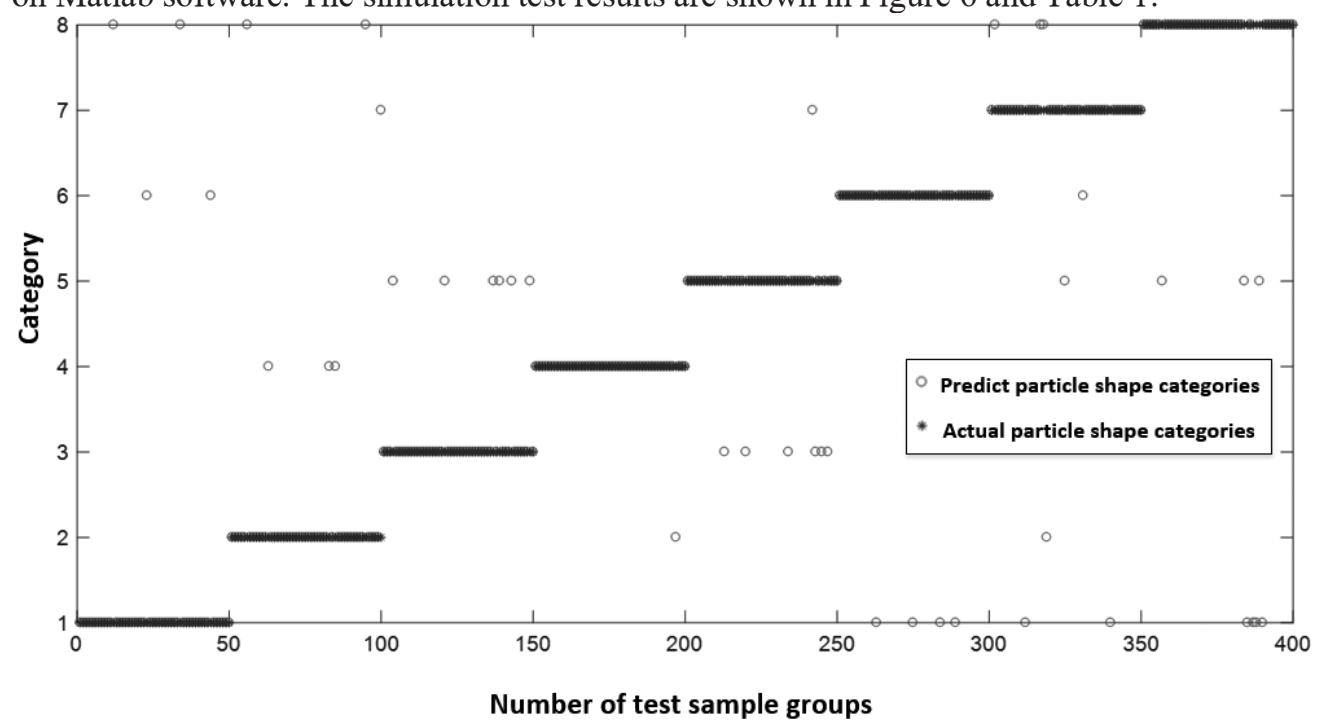

Fig. 6. Simulation prediction and actual classification comparison of the test set by BP neural network 
Table 1. BP neural network for eight types of cloud particle image shape classification recognition accuracy.

\begin{tabular}{ccccccccc}
\hline Graupel & Column & Dendrite & Needle & Aggregate & Sphere & Hexagonal & Irregular & Average recognition rate \\
\hline $92 \%$ & $88 \%$ & $88 \%$ & $98 \%$ & $86 \%$ & $92 \%$ & $84 \%$ & $86 \%$ & $89.25 \%$ \\
$(46 / 50)$ & $(44 / 50)$ & $(44 / 50)$ & $(49 / 50)$ & $(43 / 50)$ & $(46 / 50)$ & $(42 / 50)$ & $(43 / 50)$ & $(357 / 400)$ \\
\hline
\end{tabular}

In this paper, 8 types of cloud particle image shape classification accuracy rates are all above $82 \%$ and the average accuracy rate is above $88 \%$ BP neural network parameters as the subsequent BP neural network classifier for automatic classification of continuous cloud particle image fragments.

\subsection{BP neural network recognition effect verification}

In order to test the actual performance of BP neural network, part of the flight segment data measured by the detection aircraft of Shanxi Artificial Rain and Hail Prevention Office in water-dropping layer cloud in Taiyuan area on April 20, 2010 was used to compare and verify the effect of the improved Holroyd method proposed by Huang Minsong (2020) and BP neural network. The cloud particle image in the selected flight segment frame is shown in Figure 7. The range of the particle index in the frame is 42927-45190. Before performing shape recognition, it is necessary to remove pseudo particles and calculate relevant characteristic parameters. Due to the addition of new feature parameters, some particles will not be able to calculate the corresponding feature parameters. For this kind of cloud particles, the algorithm is also relatively eliminated, the number of eliminated is 61 , and the number of natural cloud particles that can be identified is 1656. The recognition results are shown in Table 2. It can be seen from the table that the BP neural network has a higher accuracy rate for cloud particle shape recognition than the improved Holroyd method, with an accuracy rate of $84.84 \%$.
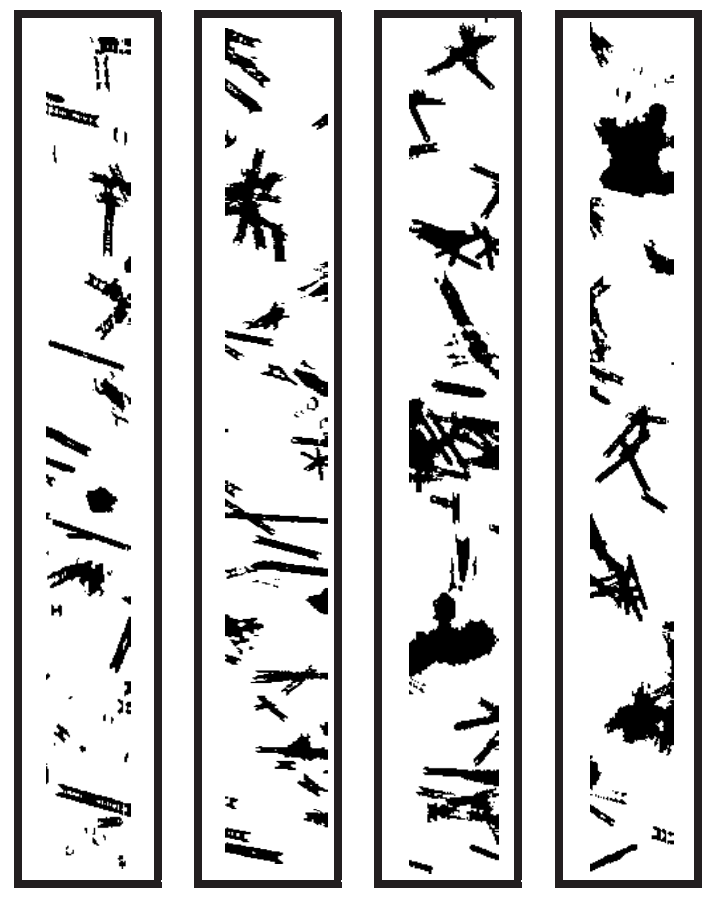

Fig. 7. Partial particle images measured by CIP. 
Table 2. Comparison of the two methods for shape recognition accuracy of BT fragments from 15:56:00 to 15:56:05 on April 20, 2010.

\begin{tabular}{lccc}
\hline & $\begin{array}{c}\text { Number of particles in frame } \\
\text { (after preprocessing) }\end{array}$ & Correctly identify particle shapes & Correct rate \\
\hline Improved Holroyd method & 1656 & 1353 & $81.70 \%$ \\
BP neural network method & 1656 & 1405 & $84.84 \%$ \\
\hline
\end{tabular}

\section{Conclusion}

This paper proposes a cloud particle shape recognition method based on BP neural network. The recognition method using BP neural network has good learning ability and fault tolerance, and its large-scale adaptive parallel processing ability meets the real-time processing requirements for large number of cloud particle images measured by CIP. It solves the trouble of manually determining different particle shape recognition thresholds for cloud particles measured by different probes and different geographical environments. In terms of actual performance, select the actual observation data segment of Taiyuan area on April 20, 2010, and select the improved Holroyd method and BP neural network method to compare the shape classification. The result proves that the BP neural network has a more obvious advantage in accuracy. The feasibility of the network to classify the shape of cloud particle images measured by CIP can provide a basis for shape recognition for subsequent research on cloud microphysics.

\section{References}

1 Guo C L. An Introduction to Atmospheric Radiation(2nd)[J]. Chinese Journal of Atmospheric Sciences, 2005(3):416.

2 Chen Q, Zhang H. Effects of ice crystal habit weight on ice cloud optical properties and radiation[J]. Acta Meteorologica Sinica, 2018,76(2):279-288.

3 Rahman M, Quincy E A, Jacquot R G, et al. Feature Extraction and Selection for Pattern Recognition of Two-Dimensional Hydrometeor Images[J]. Journal of Applied Meteorology, 1981, 20(5):521-535.

4 Rahman M, Jacquot R G, Quincy E A, et al. Two-Dimensional Hydrometeor Image Classification by Statistical Pattern Recognition Algorithms[J]. Journal of Applied Meteorology, 1981, 20(5):536-546.

5 Hunter H E, Dyer R M, Glass M. A Two-Dimensional Hydrometeor Machine Classifier Derived from Observed Data[J]. Journal of Atmospheric and Oceanic Technology, 1984, 1(1):28 36.

6 Holroyd, Edmond W. Some Techniques and Uses of 2D-C Habit Classification Software for Snow Particles[J]. Journal of Atmospheric and Oceanic Technology, 1987, 4(3):498 511.

7 Duroure C, Larsen H R, Isaka H, et al. 2D image population analysis[J]. Atmospheric Research, 1994,34(1-4): 195 205.

8 Korolev A, Sussman B. A Technique for Habit Classification of Cloud Particles[J]. Journal of Atmospheric and Oceanic Technology, 2000, 17(8):1048 1057.

9 Lawson R P, Baker B A, Zmarzly P, et al. Microphysical and Optical Properties of Atmospheric Ice Crystals at South Pole Station, Journal of Applied Meterology and Climatology, 2006, 45(11):1505 1524. 
10 Lindqvist H, Muinonen K, Nousiainen T, et al. Ice-cloud particle habit classification using principal components[J], Journal of Geophysical Research, 2012.

11 Sun H Y, Wang Z, Xu L R. A method of precipitation particle image recognition based on the moment and Fourier descriptors[J]. Chinese Journal of Stereology and Image Analysis,2014,19(1):35-43.

12 Wang L, Li C C, Zhao Z L, Yao Z G, Han Z G, Wei Q. Application of 2D habit classification in cloud microphysics analysis[J]. Chinese Journal of Atmospheric Sciences,2014,38(2):201-212.

13 Huang M S, Lei H C. An improved Holroyd cloud particle habit identification method and its application[J]. Acta Meteorologica Sinica,2020,78(2):289-300.

14 Guo J P. Analyses of Parameters and Shapes of Raindrop Size Distribution[J]. Meteorological Monthly,1996(4):41-45.

15 Huang M S, Lei H C. Playback Software of Cloud and Precipitation Particle Images Based on LabVIEW Graphic Language[J]. Meteorological Science and Technology, 2015,43(6):1060-1064.

16 Huang M S, Lei H C, Chen J T, Zhang X Q. Cloud Particle Shattering during Sampling by Airborne Optical Array Probes[J]. Chinese Journal of Atmospheric Sciences, 2016,40(3):647-656.

17 Huang M S, Lei H C, Jin L. Pseudo Particle Identification in the image data from the Airborne Cloud and Precipitation Particle Image Probe[J]. Chinese Journal of Atmospheric Sciences, 2017, 41(5):1113-1124.

18 Huang M S, Lei H C. Processing method for the partial particles and its influence on the cloud microphysical parameters measured by the airborne cloud and precipitation image probe[J]. Acta Physica Sinica,2018,67(24):285-295. 